Research Article

\title{
The Multibases of Symmetric Caterpillars
}

\section{Supachoke Isariyapalakul (D), Varanoot Khemmani (D), and Witsarut Pho-on}

Department of Mathematics, Srinakharinwirot University, Sukhumvit 23, Bangkok 10110, Thailand

Correspondence should be addressed to Varanoot Khemmani; varanoot@g.swu.ac.th

Received 2 April 2020; Accepted 6 June 2020; Published 24 June 2020

Academic Editor: Andrei V. Kelarev

Copyright (c) 2020 Supachoke Isariyapalakul et al. This is an open access article distributed under the Creative Commons Attribution License, which permits unrestricted use, distribution, and reproduction in any medium, provided the original work is properly cited.

For a set $W=\left\{w_{1}, w_{2}, \ldots, w_{k}\right\}$ of vertices and a vertex $v$ of a connected graph $G$, the $k$-multiset $m r(v \mid W)=\left\{d\left(v, w_{1}\right), d(v\right.$, $\left.\left.w_{2}\right), \ldots, d\left(v, w_{k}\right)\right\}$, where $d\left(v, w_{i}\right)$ is the distance from $v$ to $w_{i}$ for $i=1,2, \ldots, k$, and is the multirepresentation of $v$ with respect to $W$. The set $W$ is a multiresolving set of $G$ if the multirepresentations of every two distinct vertices of $G$ with respect to $W$ are distinct. The multiresolving set of $G$ having the minimum cardinality is called a multibasis of $G$. The cardinality of a multibasis of $G$ is the multidimension $\operatorname{dim}_{M}(G)$ of $G$. A caterpillar ca $\left(k_{1}, k_{2}, \ldots, k_{s}\right)$ is called a symmetric caterpillar if $k_{i}=k_{s-i+1}$ for all integers $i$ with $1 \leq i \leq s$. In this work, the multiresolving sets of symmetric caterpillars are studied.

\section{Introduction}

For an ordered set $W=\left\{w_{1}, w_{2}, \ldots, w_{k}\right\}$ of vertices and a vertex $v$ of a connected graph $G$, we refer to the following $k$-vector:

$$
r(v \mid W)=\left(d\left(v, w_{1}\right), d\left(v, w_{2}\right), \ldots, d\left(v, w_{k}\right)\right),
$$

as the representation of $v$ with respect to $W$, where $d\left(u, w_{i}\right)$ is the distance between $v$ and $w_{i}$ for $1 \leq i \leq k$. The ordered set $W$ is a resolving set of $G$ if any two distinct vertices have distinct representations with respect to $W$. A resolving set of $G$ containing a minimum number of vertices is a minimum resolving set or a basis of $G$. The dimension of $G$ is the number of vertices in a basis of $G$ and denoted by $\operatorname{dim}(G)$. To illustrate these concepts, consider a connected graph $G$ of Figure 1 with a vertex set $V(G)=\{u, v, w, x, y, z\}$.

For an ordered set $W=\{u, w\}$ based of Figure 1, the representations of vertices of $G$ with respect to $W$ are

$$
\begin{array}{lll}
r(u \mid W)=(0,3), & r(v \mid W)=(1,2), & r(w \mid W)=(3,0), \\
r(x \mid W)=(2,1), & r(y \mid W)=(3,2), & r(z \mid W)=(4,3) .
\end{array}
$$

Since the representations of two distinct vertices with respect to $W$ are distinct, it follows that $W$ is a resolving set of $G$. Using a case-by-case analysis, we can show that $G$ contains no 1-element resolving set and so $W$ is a basis of $G$. Then, $\operatorname{dim}(G)=2$.

Resolving sets have previously appeared in [1]. In [2], and later in [3], Slater introduced these ideas and used a locating set for what we have called a resolving set. He referred to the cardinality of a basis in a graph $G$ as its location number loc $(G)$. Hulme, Shiver, and Slater described in [4-6] the usefulness of these ideas when working with US sonar and coast guard Loran (long-range aids to navigation) stations. Harary and Melter [7] discovered the concept of location number independently as well but used the term of dimension. Recently, these concepts were rediscovered by Johnson [8] of the Pharmacia Company while attempting to develop a capability of large datasets of chemical graphs. More applications of these concepts to navigation of robots in networks and other areas are discussed in [9].

In general, a representation of a vertex with respect to an ordered set $W$ in a connected graph $G$ is a vector. We then consider a set $W$ of vertices of $G$ for which every vertex of $G$ has a representation with respect to $W$ in terms of a multiset.

For a set $W=\left\{w_{1}, w_{2}, \ldots, w_{k}\right\}$ of vertices and a vertex $v$ of a connected graph $G$, the multirepresentationof $v$ with respect to $W$ is a $k$-multiset:

$$
m r(v \mid W)=\left\{d\left(v, w_{1}\right), d\left(v, w_{2}\right), \ldots, d\left(v, w_{k}\right)\right\} .
$$




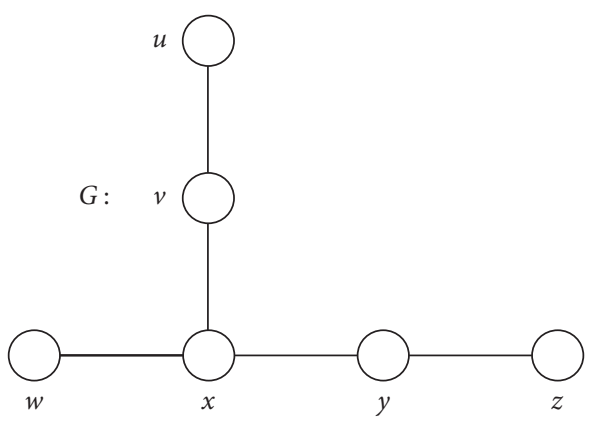

Figure 1: A connected graph G.

To emphasize that this is a multirepresentation of $v$ with respect to $W$ in $G$, we sometimes denote this multirepresentation by $m r_{G}(v \mid W)$. The set $W$ is called a multiresolving set of $G$ if every two distinct vertices have distinct multirepresentations with respect to $W$. A multiresolving set of $G$ containing a minimum cardinality is called a minimum multiresolving set or a multibasis of $G$ and this cardinality is the multidimension of $G$, which is denoted by $\operatorname{dim}_{M}(G)$.

To illustrate these concepts, consider a connected graph $G$ of Figure 1 . We now know that the set $W=\{u, w\}$ is a resolving set of $G$. However, since $\operatorname{mr}(u \mid W)=\{0,3\}=$ $m r(w \mid W)$, it follows that $W$ is not a multiresolving set of $G$. On the other hand, the set $W^{\prime}=\{u, v, z\}$ is a multiresolving set of $G$ since the multirepresentations of vertices of $G$ with respect to $W^{\prime}$ are

$$
\begin{array}{ll}
m r\left(u \mid W^{\prime}\right)=\{0,1,4\}, & \operatorname{mr}\left(v \mid W^{\prime}\right)=\{0,1,3\}, \quad \operatorname{mr}\left(w \mid W^{\prime}\right)=\{2,3,3\} \\
m r\left(x \mid W^{\prime}\right)=\{1,2,2\}, & \operatorname{mr}\left(y \mid W^{\prime}\right)=\{1,2,3\}, \quad \operatorname{mr}\left(z \mid W^{\prime}\right)=\{0,3,4\}
\end{array}
$$

Since $G$ contains neither 1-element nor 2-element multiresolving set of $G$, it follows that $W^{\prime}$ is a multibasis of $G$; that is, $\operatorname{dim}_{M}(G)=3$.

For $n \geq 3$, the complete graph $K_{n}$ contains no multiresolving set; that is, $\operatorname{dim}_{M}\left(K_{n}\right)$ is not defined which is described in [10]. In fact, $\operatorname{dim}_{M}(G)$ is not defined for all connected graphs $G$. However, for a connected graph $G$ of order $n$ such that $\operatorname{dim}_{M}(G)$ is defined, every multiresolving set of $G$ is also a resolving set of $G$, and so,

$$
1 \leq \operatorname{dim}(G) \leq \operatorname{dim}_{M}(G) \leq n .
$$

For a set $W$ of vertices of a connected graph $G$, the multirepresentation of vertices of $W$ with respect to $W$ contains 0 . On the other hand, the vertices of $G$ whose multirepresentations with respect to $W$ do not contain 0 are vertices in $V(G)-W$. Therefore, to determine whether a set $W$ is a multiresolving set of $G$, it suffices to show that every two distinct vertices in $W$ have distinct multirepresentations with respect to $W$ and then determine that multirepresentations with respect to $W$ of all vertices in $V(G)-$ $W$ are distinct.

The concepts of multiresolving set and multidimension were introduced in [10] and further studied in [11-13].

\section{Preliminaries}

For two vertices $u$ and $v$ of a connected graph $G$, if $d(u, x)=d(v, x)$ for all $x \in V(G)-\{u, v\}$, then $u$ and $v$ are distance-similar. Distance similarity is an equivalence relation on $V(G)$. For example, consider the cycle $C_{4}=\left(v_{1}, v_{2}, v_{3}, v_{4}\right)$. There are two distance-similar equivalence classes, one that is $\left\{v_{1}, v_{3}\right\}$ and one that is $\left\{v_{2}, v_{4}\right\}$. The distance-similar equivalence class is useful to verify the multiresolving set of a connected graph as the following results.
Theorem 1 (see [10]). Let $G$ be a connected graph such that $\operatorname{dim}_{M}(G)$ is defined. If $U$ is a distance-similar equivalence class in $G$ with $|U|=2$, then every multiresolving set of $G$ contains exactly one vertex of $U$.

Theorem 2 (see [10]). If $U$ is a distance-similar equivalence class in a connected graph $G$ with $|U| \geq 3$, then $\operatorname{dim}_{M}(G)$ is not defined.

It was shown in $[10,13]$ that a path $P_{n}=\left(u_{1}, u_{2}, \ldots, u_{n}\right)$ is the only connected graph with multidimension 1 and $\left\{u_{1}\right\}$ and $\left\{u_{n}\right\}$ are the only two multibases of $P_{n}$ for $n \geq 2$. There is no connected graph with multidimension 2 . We state these results in the next theorems.

Theorem 3 (see $[10,13])$. Let $G$ be a connected graph. Then, $\operatorname{dim}_{M}(G)=1$ if and only if $G=P_{n}$, the path of order $n$.

Theorem 4 (see $[10,13])$. A connected graph has no multiresolving set of cardinality 2.

The following useful results for finding a multiresolving set of a connected graph were described in [12].

Theorem 5 (see [12]). Let $G$ be a connected graph such that $\operatorname{dim}_{M}(G)$ is defined, and let $W$ be a multiresolving set of $G$. If $v$ is an end-vertex of $G$ such that $v \notin W$, then $W$ is a multiresolving set of $G-v$.

Theorem 6 (see [12]). Let $G$ be a connected graph such that $\operatorname{dim}_{M}(G)$ is defined, and let $W$ be a multiresolving set of $G$. If $v_{1}, v_{2}, \ldots, v_{t}$ are end-vertices of $G$ such that $v_{1}, v_{2}, \ldots$, $v_{t} \notin W$, then $W$ is a multiresolving set of $G-\left\{v_{1}, v_{2}, \ldots, v_{t}\right\}$.

Theorem 7 (see [12]). Let $T$ be a tree of order at least 3 containing a vertex $u$. If $W$ is a multiresolving set of $T$, then $W$ 
contains at least one vertex from each of $\operatorname{deg}_{T} u$ components of $T-u$, with one possible exception.

\section{Multibases of Symmetric Caterpillars}

A caterpillar is a tree of order at least 3 in which the removal of all end-vertices results in a path. This path is called the spine of a caterpillar. A vertex of the spine of a caterpillar is called a spine-vertex. Let $T$ be a caterpillar such that $\operatorname{dim}_{M}(T)$ is defined. Since any two end-vertices that are adjacent to the same spine-vertex of $T$ are distance-similar, it follows from Theorem 2 that there are at most two end-vertices being adjacent to each spine-vertex of $T$. Therefore, we consider multibases of such a caterpillar $T$. In order to do this, some additional definitions and notation are introduced. For integers $s, k_{1}, k_{2}, \ldots, k_{s}$ with $s \geq 1, \quad 1 \leq k_{1}, k_{s} \leq 2$, and $0 \leq k_{2}, k_{3}, \ldots, k_{s-1} \leq 2$, let $\mathrm{ca}\left(k_{1}, k_{2}, \ldots, k_{s}\right)$ be a caterpillar which is obtained from the spine $\left(u_{1}, u_{2}, \ldots, u_{s}\right)$ by joining $k_{i}$ end-vertices to the spine-vertex $u_{i}$, where $1 \leq i \leq s$. Observe that if $k_{i}=0$, then there is no end-vertex joining to the spinevertex $u_{i}$. Also, if $k_{i}=1$, then the spine-vertex $u_{i}$ is adjacent to an end-vertex which is called the first end-vertex $v_{i}$ of $u_{i}$. Furthermore, if $k_{i}=2$, then there are two end-vertices joining to $u_{i}$ that are called the first and second end-vertices of $u_{i}$ and denoted by $v_{i}$ and $w_{i}$, respectively. For each integer $i$ with $1 \leq i \leq s$, we define the set $\Psi=\left\{i \in \mathbb{Z} \mid k_{i}=2\right\}$ to be the second end-set of a caterpillar $\mathrm{ca}\left(k_{1}, k_{2}, \ldots, k_{s}\right)$. To emphasize that this is the second end-set $\Psi$ of a caterpillar $T$, we sometimes denote this set by $\Psi_{T}$. The caterpillar ca $(1,2,0,2,1,2,2)$ with the second end-set $\Psi=\{2,4,6,7\}$ is illustrated in Figure 2 .

The caterpillars having multidimension 3 were studied and characterized in [12]. This suggests a way of investigating caterpillars having the multidimension of at least 3. Notice that the multidimension of a caterpillar is established by its second end-set $\Psi$. For a caterpillar $\mathrm{ca}\left(k_{1}, k_{2}, \ldots, k_{s}\right)$, observe that for each $i \in \Psi$, if $s-i+1 \in \Psi$, then the multirepresentations of second end-vertices $w_{i}$ and $w_{s-i+1}$ with respect to the set $W=\left\{w_{i} \mid i \in \Psi\right\}$ are the same. This leads us to determine a multibasis of a particular caterpillar. In order to do this, we need an additional definition. A caterpillar $\mathrm{ca}\left(k_{1}, k_{2}, \ldots, k_{s}\right)$ is called a symmetric caterpillar if $k_{i}=k_{s-i+1}$ for each integer $i$ with $1 \leq i \leq s$. For instance, the symmetric caterpillar $\mathrm{ca}(2,0,2,1,2,0,2)$ is shown in Figure 3.

For $s=1$, the symmetric caterpillar $\mathrm{ca}(2)$ is a path. Theorem 3 implies that its multidimension is 1 with multibases $\left\{v_{1}\right\}$ and $\left\{w_{1}\right\}$. For $s=2$, there are two symmetric caterpillars $\mathrm{ca}(1,1)$ and $\mathrm{ca}(2,2)$. Indeed, $\mathrm{ca}(1,1)$ is a path whose multidimension is 1 with a multibasis $\left\{v_{1}\right\}$. It is routine to verify that the multidimension of $\mathrm{ca}(2,2)$ is 3 with a multibasis $\left\{u_{1}, w_{1}, w_{2}\right\}$. Multibases of these caterpillars are indicated in Figure 4 by solid vertices.

As mentioned earlier, the multidimension of a path is 1 . We may therefore consider symmetric caterpillars that are not a path. For $s \geq 3$, let $T$ be a symmetric caterpillar $\mathrm{ca}\left(k_{1}, k_{2}, \ldots, k_{s}\right)$ that is not a path. If $|\Psi|=0$, then $T$ is a symmetric caterpillar ca $\left(k_{1}, k_{2}, \ldots, k_{s}\right)$ with $k_{r}=1$ for some $r \in\{2,3, \ldots, s-1\}$. It is shown in [12] that a set $\left\{u_{1}, v_{1}, v_{s}\right\}$ is a multibasis of $T$, and so $\operatorname{dim}_{M}(T)=3$. If $|\Psi|=1$, then there is only one integer $i$ belonging to $\Psi$ for some $i \in\{1,2, \ldots, s\}$.

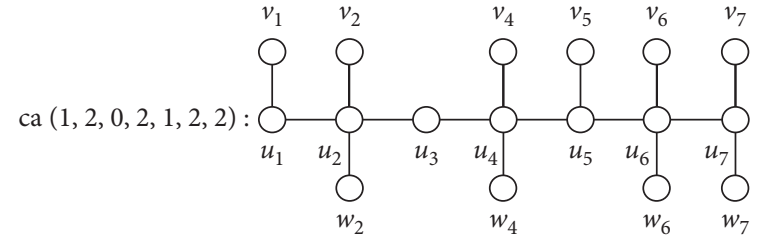

Figure 2: The caterpillar ca $(1,2,0,2,1,2,2)$ with $\Psi=\{2,4,6,7\}$.

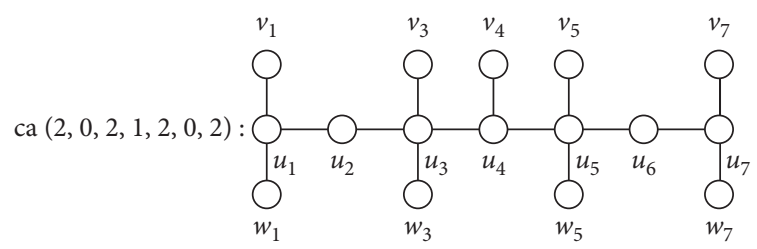

Figure 3: The symmetric caterpillar ca $(2,0,2,1,2,0,2)$

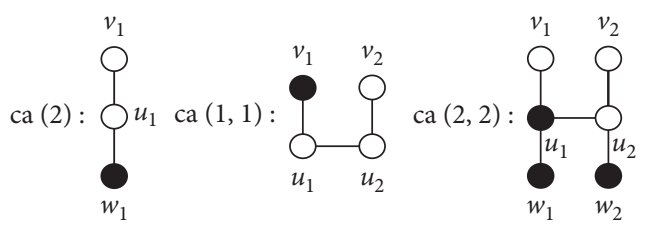

Figure 4: The symmetric caterpillar ca(2), ca $(1,1)$, and $\mathrm{ca}(2,2)$

Since $T$ is a symmetric caterpillar, it follows that $i=s-i+1$, and so $i=(s+1) / 2$. This implies that $s$ is odd and $\Psi=\{(s+1) / 2\}$. Thus, there are two possibilities: (1) $s=3$ or (2) $s>3$. For $s=3$, the symmetric caterpillar $T \cong \mathrm{ca}(1,2,1)$ has multidimension 4 with a multibasis $\left\{u_{1}, v_{1}, v_{3}, w_{2}\right\}$. For $s>3$, a symmetric caterpillar $T$ has multidimension 3 with a multibasis $\left\{u_{1}, v_{s}, w_{(s+1) / 2}\right\}$. We therefore investigate a multibasis and the multidimension of a symmetric caterpillar ca $\left(k_{1}, k_{2}, \ldots, k_{s}\right)$ with $|\Psi| \geq 2$, where $s \geq 3$. The following useful results are shown in [12], as we state next.

Proposition 1 (see [12]). Let $s, \alpha$, and $\beta$ be integers with $s \geq 3$ and $1 \leq \alpha<\beta \leq s$, and let $W$ be a set of vertices of a caterpillar $c a\left(k_{1}, k_{2}, \ldots, k_{s}\right)$ containing one of $\left\{v_{1}, w_{1}\right\}$ and one of $\left\{v_{s}, w_{s}\right\}$. If $m r\left(u_{\alpha} \mid W\right)=m r\left(u_{\beta} \mid W\right)$ or $m r\left(v_{\alpha} \mid W\right)=$ $m r\left(v_{\beta} \mid W\right)$, then $1 \leq \alpha \leq\lceil s / 2\rceil$ and $\beta=s-\alpha+1$.

Proposition 2 (see [12]). Let $s, \gamma$, and $\delta$ be integers with $s \geq 3$ and $1 \leq \gamma$ and $\delta \leq s$, and let $W$ be a set of vertices of a caterpillar ca $\left(k_{1}, k_{2}, \ldots, k_{s}\right)$ containing one of $\left\{v_{1}, w_{1}\right\}$ and one of $\left\{v_{s}, w_{s}\right\}$. Then,

(i) If $1 \leq \gamma<\delta \leq s$ and $m r\left(v_{\gamma} \mid W\right)=m r\left(u_{\delta} \mid W\right)$, then $1 \leq \gamma \leq\lceil s / 2\rceil$ and $\delta=s-\gamma+2$

(ii) If $1 \leq \delta \leq \gamma \leq s$ and $m r\left(v_{\gamma} \mid W\right)=m r\left(u_{\delta} \mid W\right)$, then $\lceil s / 2\rceil+1 \leq \gamma \leq s$ and $\delta=s-\gamma$

Proposition 3 (see [12]). Let $s, \alpha$, and $\beta$ be integers with $s \geq 3$ and $1 \leq \alpha<\beta \leq s$, and let $W$ be a set of vertices of a caterpillar $c a\left(k_{1}, k_{2}, \ldots, k_{s}\right)$ containing $u_{1}$ and one of $\left\{v_{s}, w_{s}\right\}$ except $v_{1}$ 
and $w_{1}$. If $m r\left(u_{\alpha} \mid W\right)=m r\left(u_{\beta} \mid W\right)$ or $\operatorname{mr}\left(v_{\alpha} \mid W\right)=$ $\operatorname{mr}\left(v_{\beta} \mid W\right)$, then $1 \leq \alpha \leq\lceil s / 2\rceil$ and $\beta=s-\alpha+2$.

Proposition 4 (see [12]). Let $s, \gamma$, and $\delta$ be integers with $s \geq 3$ and $1 \leq \gamma, \delta \leq s$, and let $W$ be a set of vertices of a caterpillar ca $\left(k_{1}, k_{2}, \ldots, k_{s}\right)$ containing $u_{1}$ and one of $\left\{v_{s}, w_{s}\right\}$ except $v_{1}$ and $w_{1}$. Then,

(i) If $1 \leq \gamma<\delta \leq s$ and $m r\left(v_{\gamma} \mid W\right)=m r\left(u_{\delta} \mid W\right)$, then $1 \leq \gamma \leq\lceil s / 2\rceil$ and $\delta=s-\gamma+3$

(ii) If $1 \leq \delta \leq \gamma \leq s$ and $m r\left(v_{\gamma} \mid W\right)=m r\left(u_{\delta} \mid W\right)$, then $\lceil s / 2\rceil+1 \leq \gamma \leq s$ and $\delta=s-\gamma+1$

The following observation is a consequence of Theorem 1.

Observation 1. Every multiresolving set of a caterpillar $\mathrm{ca}\left(k_{1}, k_{2}, \ldots, k_{s}\right)$ with a second end-set $\Psi$ contains either first end-vertex $v_{i}$ or second end-vertex $w_{i}$, where $i \in \Psi$.

By Observation 1, the multidimension of a symmetric caterpillar ca $\left(k_{1}, k_{2}, \ldots, k_{s}\right)$ with a second end-set $\Psi$ must be at least $|\Psi|$. In fact, its multidimension is at least $|\Psi|+1$, as we now show.

Proposition 5. For $s \geq 3$, let $T$ be a symmetric caterpillar $c a\left(k_{1}, k_{2}, \ldots, k_{s}\right)$ such that $\operatorname{dim}_{M}(T)$ is defined with the second end-set $\Psi$. Then, $\operatorname{dim}_{M}(T) \geq|\Psi|+1$.

Proof. If $|\Psi|=0$, then the result holds. We may assume for $|\Psi| \geq 1$ that the statement of the proposition is false. Then, there is a symmetric caterpillar ca $\left(k_{1}, k_{2}, \ldots, k_{s}\right)$ having a multiresolving set $W$ with $|W| \leq|\Psi|$. By Observation 1, $|W|=|\Psi|$. However, then, $m r\left(u_{1} \mid W\right)=m r\left(u_{s} \mid W\right)$, contradicting $W$ as being a multiresolving set of $\mathrm{ca}\left(k_{1}\right.$, $\left.k_{2}, \ldots, k_{s}\right)$.

Proposition 5 states that $|\Psi|+1$ is a lower bound for the multidimension of a symmetric caterpillar. Furthermore, we also establish that $|\Psi|+3$ is an upper bound for the multidimension of a symmetric caterpillar, as follows.

Proposition 6. For $s \geq 3$, let $T$ be a symmetric caterpillar ca $\left(k_{1}, k_{2}, \ldots, k_{s}\right)$ such that $\operatorname{dim}_{M}(T)$ is defined with a second end-set $\Psi$. Then, $\operatorname{dim}_{M}(T) \leq|\Psi|+3$.

Proof. To show $\operatorname{dim}_{M}(T) \leq|\Psi|+3$, it suffices to verify that there is a multiresolving set of $T$ having cardinality at most $|\Psi|+3$. Let $W$ be the set of all second end-vertices of $T$ with $|W|=|\Psi|$. We consider three cases for $\Psi$.

Case 1. 1 belongs to $\Psi$.

We claim that $B=W \cup\left\{u_{1}\right\}$ is a multiresolving set of $T$. Assume, contrary to our claim, that there are two vertices $x$ and $y$ of $T$ such that $m r(x \mid B)=m r(y \mid B)$. We consider two subcases.

Subcase 1.1. Both $x$ and $y$ belong to $B$.

First, we show that both $x$ and $y$ belong to $W$. Suppose, to the contrary, that either $x$ or $y$ does not belong to $W$, say $x$. Then, $x=u_{1}$. Since $u_{1}$ and $w_{1}$ are the only two adjacent vertices of $B$, it follows that $\operatorname{mr}\left(u_{1} \mid B\right)$ contains 1 , and so $y=w_{1}$. However, since $d\left(u_{1}, w_{s}\right)$ and $d\left(w_{1}, w_{s}\right)$ are the maximum elements of $m r\left(u_{1} \mid B\right)$ and $m r\left(w_{1} \mid B\right)$, respectively, it follows that $d\left(u_{1}, w_{s}\right)=$ $d\left(w_{1}, w_{s}\right)$, which is a contradiction. Therefore, both $x$ and $y$ must belong to $W$. Next, we let $x=w_{\alpha}$ and $y=w_{\beta}$, where $1 \leq \alpha<\beta \leq s$. If $1 \leq \alpha<\beta \leq\lceil s / 2\rceil$, then $d\left(w_{\alpha}, w_{s}\right)=s-\alpha+2$ and $d\left(w_{\beta}, w_{s}\right)=s-\beta+2$ are the maximum elements of $m r\left(w_{\alpha} \mid B\right)$ and $m r\left(w_{\beta} \mid B\right)$, respectively. Therefore, $\alpha=\beta$, producing a contradiction. Similarly, if $\lceil s / 2\rceil+1 \leq \alpha<\beta \leq s$, then $d\left(w_{\alpha}, w_{1}\right)=$ $\alpha+1$ and $d\left(w_{\beta}, w_{1}\right)=\beta+1$ must be equal, that is, $\alpha=\beta$, which is impossible. We may assume that $1 \leq \alpha \leq\lceil s / 2\rceil$ and $\lceil s / 2\rceil+1 \leq \beta \leq s$. Since $d\left(w_{\alpha}, w_{s}\right)=s-$ $\alpha+2$ and $d\left(w_{\beta}, w_{1}\right)=\beta+1$ are the maximum elements of $m r\left(w_{\alpha} \mid B\right)$ and $m r\left(w_{\beta} \mid B\right)$, respectively, it follows that $\beta=s-\alpha+1$. Since $T$ is a symmetric caterpillar, it follows that $m r\left(w_{\alpha} \mid W\right)=m r\left(w_{\beta} \mid W\right)$. However, since $d\left(w_{\alpha}, u_{1}\right)<d\left(w_{\beta}, u_{1}\right)$, it follows that $m r\left(w_{\alpha} \mid B\right) \neq$ $m r\left(w_{\beta} \mid B\right)$, this contradicts our assumption.

Subcase 1.2. Neither $x$ nor $y$ belongs to $B$.

We consider three subcases.

Subcase 1.2.1. $x$ and $y$ are spine-vertices.

Let $x=u_{\alpha}$ and $y=u_{\beta}$, where $2 \leq \alpha<\beta \leq s$. Applying Proposition 1, we obtain that $2 \leq \alpha \leq\lceil s / 2\rceil$ and $\beta=s-\alpha+1$. Since $m r\left(u_{\alpha} \mid W\right)=m r\left(u_{s-\alpha+1} \mid W\right)$ and $d\left(u_{\alpha}, u_{1}\right)<d\left(u_{s-\alpha+1}, u_{1}\right)$, it follows that $\operatorname{mr}\left(u_{\alpha} \mid B\right) \neq$ $m r\left(u_{\beta} \mid B\right)$, which is a contradiction.

Subcase 1.2.2. $x$ and $y$ are first end-vertices.

Let $x=v_{\alpha}$ and $y=v_{\beta}$, where $1 \leq \alpha<\beta \leq s$. By Proposition $1, \quad 1 \leq \alpha \leq\lceil s / 2\rceil$ and $\beta=s-\alpha+1$. Since $m r\left(v_{\alpha} \mid W\right)=m r\left(v_{s-\alpha+1} \mid W\right)$ and $d\left(v_{\alpha}, u_{1}\right)<d\left(v_{s-\alpha+1}\right.$, $\left.u_{1}\right)$, it follows that $m r\left(v_{\alpha} \mid B\right) \neq m r\left(v_{\beta} \mid B\right)$, this is also a contradiction.

Subcase 1.2.3. $x$ is a first end-vertex and $y$ is a spinevertex.

If $\Psi=\{1, s\}$, then it is shown in [12] that the set $\left\{u_{1}, w_{1}, w_{s}\right\}$ is a multiresolving set of $T$. We therefore consider the second end-set of cardinality at least 3 . Let $p=\min (\Psi-\{1, s\})$. By the symmetry of $T, s-p+$ $1=\max (\Psi-\{1, s\})$. Let $x=v_{\gamma}$ and $y=u_{\delta}$, where $1 \leq$ $\gamma, \delta \leq s$. We consider two subcases for $\gamma$ and $\delta$.

Subcase 1.2.3.1. $1 \leq \gamma<\delta \leq s$.

By Proposition 2 (i), $1 \leq \gamma \leq\lceil s / 2\rceil$ and $\delta=s-\gamma+2$. Since $d\left(v_{\gamma}, w_{s}\right)$ and $d\left(u_{\delta}, w_{1}\right)$ are the maximum elements of $m r\left(v_{\gamma} \mid B\right)$ and $m r\left(u_{\delta} \mid B\right)$, respectively, it follows that $\max \left(\operatorname{mr}\left(v_{\gamma} \mid B-\left\{w_{s}\right\}\right)\right)=d\left(v_{\gamma}, w_{s-p+1}\right)=$ $s-p-\gamma+3$ and $\max \left(\operatorname{mr}\left(u_{\delta} \mid B-\left\{w_{1}\right\}\right)\right)=d\left(u_{\delta}, u_{1}\right)$ $=\delta-1$ are the same. Consequently, $p=2$ and so $\delta-1=s-\gamma+1$. Since $d\left(u_{\delta}, u_{1}\right)$ and $d\left(u_{\delta}, w_{2}\right)$ are in $m r\left(u_{\delta} \mid B\right)$, it follows that $m r\left(v_{\gamma} \mid B\right)$ also contains two $(s-\gamma+1) s$. Notice that $u_{s}, v_{s-1}$, and $w_{s-1}$ are the only three vertices of $T$ whose distance from $v_{\gamma}$ is $s-\gamma+1=\delta-1$. Since $u_{s}$ and $v_{s-1}$ do not belong to $B$, it follows that $m r\left(v_{\gamma} \mid B\right)$ contains only one element of $s-\gamma+1$, which contradicts our assumption.

Subcase 1.2.3.2. $2 \leq \delta \leq \gamma \leq s$. 
Applying Proposition 2 (ii), we obtain that $\lceil s / 2\rceil+$ $1 \leq \gamma \leq s$ and $\delta=s-\gamma$. Since $d\left(v_{\gamma}, w_{1}\right)$ and $d\left(u_{\delta}, w_{s}\right)$ are the maximum elements of $\operatorname{mr}\left(v_{\gamma} \mid B\right)$ and $m r\left(u_{\delta} \mid B\right)$, respectively, it follows that $\max (m r$ $\left.\left(v_{\gamma} \mid B-\left\{w_{1}\right\}\right)\right)=d\left(v_{\gamma}, u_{1}\right)=\gamma$ and $\max \left(\operatorname{mr}\left(u_{\delta} \mid B-\right.\right.$ $\left.\left.\left\{w_{s}\right\}\right)\right)=d\left(u_{\delta}, w_{s-p+1}\right)=s-p-\delta+2$ are the same. Certainly, $p=2$ and so $\gamma=s-\delta$. Since $d\left(v_{\gamma}, u_{1}\right)$ and $d\left(v_{\gamma}, w_{2}\right)$ are in $m r\left(v_{\gamma} \mid B\right)$, it follows that $m r\left(u_{\delta} \mid B\right)$ also contains two $(s-\delta)$ s. Notice that $u_{s}, v_{s-1}$, and $w_{s-1}$ are the only three vertices of $T$ whose distance from $u_{\delta}$ is $s-\delta=\gamma$. Since $u_{s}$ and $v_{s-1}$ do not belong to $B$, it follows that $m r\left(u_{\delta} \mid B\right)$ contains only one element of $s-\delta$, which contradicts our assumption.

Hence, in Subcases 1.1 and 1.2 above, $m r(x \mid B) \neq m r(y \mid B)$ for all $x, y \in V(T)$. This implies that $B$ is a multiresolving set of $T$.

Case 2. 1 and 2 do not belong to $\Psi$.

Symmetrically, $s-1$ and $s$ also do not belong to $\Psi$. Let $p=\min (\Psi)$. Then, $s-p+1=\max (\Psi)$. We claim that $B=$ $W \cup\left\{u_{1}, v_{s}\right\}$ is a multiresolving set of $T$. Suppose, contrary to our claim, that there are two vertices $x$ and $y$ such that $m r(x \mid B)=m r(y \mid B)$. We consider two subcases.

Subcase 2.1. Both $x$ and $y$ belong to $B$.

We first show that $u_{1}, v_{s} \notin\{x, y\}$. Assume, to the contrary, that $u_{1}=x$. Since $\max \left(\operatorname{mr}\left(u_{1} \mid B\right)\right)=$ $d\left(u_{1}, v_{s}\right)=\max (\operatorname{mr}(y \mid B))$, it follows that $y=v_{s}$. However, since $d\left(u_{1}, u_{1}\right)$ and $d\left(v_{s}, v_{s}\right)$ are the minimum elements of $m r\left(u_{1} \mid B\right)$ and $m r\left(v_{s} \mid B\right)$, respectively, and clearly, $\min \left(\operatorname{mr}\left(u_{1} \mid B-\left\{u_{1}\right\}\right)\right)=d\left(u_{1}\right.$, $\left.w_{p}\right)=p$ and $\min \left(\operatorname{mr}\left(v_{s} \mid B-\left\{v_{s}\right\}\right)\right)=d\left(v_{s}, w_{s-p+1}\right)=$ $p-1$, it follows that $m r\left(u_{1} \mid B\right) \neq m r\left(v_{s} \mid B\right)$, producing a contradiction. Thus, $x$ and $y$ belong to $W$. Next, we let $x=w_{\alpha}$ and $y=w_{\beta}$, where $p \leq \alpha<\beta \leq s-p+1$. If $1 \leq \alpha<\beta \leq\lceil s / 2\rceil$, then $d\left(w_{\alpha}, v_{s}\right)$ and $d\left(w_{\beta}, v_{s}\right)$ are the maximum elements of $m r\left(w_{\alpha} \mid B\right)$ and $m r\left(w_{\beta} \mid B\right)$, respectively. Therefore, $\alpha=\beta$, which is a contradiction. Similarly, if $\lceil s / 2\rceil+1 \leq \alpha<\beta \leq s$, then $d\left(w_{\alpha}, u_{1}\right)$ and $d\left(w_{\beta}, u_{1}\right)$ must be equal, which is impossible. We may assume that $1 \leq \alpha \leq\lceil s / 2\rceil$ and $\lceil s / 2\rceil+1 \leq \beta \leq s$. Since $d\left(w_{\alpha}, v_{s}\right)$ and $d\left(w_{\beta}, u_{1}\right)$ are the maximum elements of $m r\left(w_{\alpha} \mid B\right)$ and $m r\left(w_{\beta} \mid B\right)$, respectively, it follows that $\beta=s-\alpha+2$. Since $\max \left(\operatorname{mr}\left(w_{\alpha} \mid B-\left\{v_{s}\right\}\right)\right)=d\left(w_{\alpha}\right.$, $\left.w_{s-p+1}\right)=s-p-\alpha+3$ and $\max \left(\operatorname{mr}\left(w_{\beta} \mid B-\left\{u_{1}\right\}\right)\right)=$ $d\left(w_{\beta}, w_{p}\right)=\beta-p+2$ are equal, it follows that, certainly, $\beta=s-\alpha+1$, producing a contradiction.

Subcase 2.2. Neither $x$ nor $y$ belongs to $B$.

We consider three subcases.

Subcase 2.2.1. $x$ and $y$ are spine-vertices.

Let $x=u_{\alpha}$ and $y=u_{\beta}$, where $2 \leq \alpha<\beta \leq s$. By applying Proposition 3, it implies that $2 \leq \alpha \leq\lceil s / 2\rceil$ and $\beta=$ $s-\alpha+2$. Since $d\left(u_{\alpha}, v_{s}\right)$ and $d\left(u_{\beta}, u_{1}\right)$ are the maximum elements of $m r\left(u_{\alpha} \mid B\right)$ and $m r\left(u_{\beta} \mid B\right)$, respectively, it follows that $\max \left(\operatorname{mr}\left(u_{\alpha} \mid B-\left\{v_{s}\right\}\right)\right)=d\left(u_{\alpha}\right.$, $\left.w_{s-p+1}\right)=s-p-\alpha+2$ and $\max \left(m r\left(u_{\beta} \mid B-\left\{u_{1}\right\}\right)\right)=$ $d\left(u_{\beta}, w_{p}\right)=\beta-p+1$ must be equal. Necessarily, then, $\beta=s-\alpha+1$. This is a contradiction.

Subcase 2.2.2. $x$ and $y$ are first end-vertices.

Let $x=v_{\alpha}$ and $y=v_{\beta}$, where $1 \leq \alpha<\beta \leq s$. By Proposition $3,1 \leq \alpha \leq\lceil s / 2\rceil$ and $\beta=s-\alpha+2$. Since $d\left(v_{\alpha}, v_{s}\right)$ and $d\left(v_{\beta}, u_{1}\right)$ are the maximum elements of $m r\left(v_{\alpha} \mid B\right)$ and $m r\left(v_{\beta} \mid B\right)$, respectively, it follows that $\max \left(m r\left(v_{\alpha} \mid B-\left\{v_{s}\right\}\right)\right)=d\left(v_{\alpha}, w_{s-p+1}\right)=s-p-\alpha+3$ and $\max \left(\operatorname{mr}\left(v_{\beta} \mid B-\left\{u_{1}\right\}\right)\right)=d\left(v_{\beta}, w_{p}\right)=\beta-p+2$ must be equal. Consequently, $\beta=s-\alpha+1$. This is also a contradiction.

Subcase 2.2.3. $x$ is a first end-vertex and $y$ is a spinevertex.

Let $x=v_{\gamma}$ and $y=u_{\delta}$, where $1 \leq \gamma, \delta \leq s$. We consider two subcases according to $\gamma$ and $\delta$.

Subcase 2.2.3.1. $1 \leq \gamma<\delta \leq s$.

By Proposition 4 (i), $1 \leq \gamma \leq\lceil s / 2\rceil$ and $\delta=s-\gamma+3$. Since $d\left(v_{\gamma}, v_{s}\right)$ and $d\left(u_{\delta}, u_{1}\right)$ are the maximum elements of $m r\left(v_{\gamma} \mid B\right)$ and $m r\left(u_{\delta} \mid B\right)$, respectively, it follows that $\max \left(\operatorname{mr}\left(v_{\gamma} \mid B-\left\{v_{s}\right\}\right)\right)=d\left(v_{\gamma}, w_{s-p+1}\right)=$ $s-p-\gamma+3$ and $\max \left(\operatorname{mr}\left(u_{\delta} \mid B-\left\{u_{1}\right\}\right)\right)=d\left(u_{\delta}\right.$, $\left.w_{p}\right)=p-\delta+1$ must be equal. Evidently, $\delta=s-\gamma+2$, which is impossible.

Subcase 2.2.3.2. $2 \leq \delta \leq \gamma \leq s$.

Applying Proposition 4 (ii), it implies that $\lceil s / 2\rceil+1 \leq \gamma \leq s$ and $\delta=s-\gamma+1$. Since $d\left(v_{\gamma}, u_{1}\right)$ and $d\left(u_{\delta}, v_{s}\right)$ are the maximum elements of $\operatorname{mr}\left(v_{\gamma} \mid B\right)$ and $m r\left(u_{\delta} \mid B\right)$, respectively, it follows that $\max \left(m r\left(v_{\gamma} \mid B-\right.\right.$ $\left.\left.\left\{u_{1}\right\}\right)\right)=d\left(v_{\gamma}, w_{p}\right)=\gamma-p+2$ and $\max \left(\operatorname{mr}\left(u_{\delta} \mid B-\left\{v_{s}\right\}\right)\right)=$ $d\left(u_{\delta}, w_{s-p+1}\right)=s-p-\delta+2$ are equal. As verified above, $\delta=s-\gamma$, which cannot occur.

Hence, $m r(x \mid B) \neq m r(y \mid B)$ for all $x, y \in V(T)$. This implies that $B$ is a multiresolving set of $T$.

Case 3. 1 does not belong to $\Psi$ and 2 belongs to $\Psi$.

Let $T^{\prime}$ be a symmetric caterpillar which is obtained from $T$ by joining end-vertices $x$ and $y$ to the spine-vertices $u_{1}$ and $u_{s}$, respectively. Thus, $1 \in \Psi_{T^{\prime}}$. By applying Case $1, B=$ $\left(W \cup\left\{v_{1}, v_{s}\right\}\right) \cup\left\{u_{1}\right\}$ is a multiresolving set of $T^{\prime}$. Since $T \cong T^{\prime}-\{x, y\}$, it follows by Theorem 6 that $B=W \cup$ $\left\{u_{1}, v_{1}, v_{s}\right\}$ is a multiresolving set of $T$.

Hence, every symmetric caterpillar $T$ has a multiresolving set of cardinality of at most $|\Psi|+3$, and so $\operatorname{dim}_{M}(T) \leq|\Psi|+3$.

The following result is obtained from the bounds given in Propositions 5 and 6.

Corollary 1. For $s \geq 3$, let $T$ be a symmetric caterpillar $c a\left(k_{1}, k_{2}, \ldots, k_{s}\right)$ with the second end-set $\Psi$. Then,

$$
|\Psi|+1 \leq \operatorname{dim}_{M}(T) \leq|\Psi|+3 .
$$

The multidimensions of symmetric caterpillars are characterized by the following result. Furthermore, the sharpnesses of Corollary 1 are presented. 
Theorem 8. For $s \geq 3$, let $T$ be a symmetric caterpillar ca $\left(k_{1}, k_{2}, \ldots, k_{s}\right)$ with $|\Psi| \geq 2$ and let $W$ be a set of all second end-vertices. Then,

(i) If $1 \in \Psi$, then $W \cup\left\{u_{1}\right\}$ is a multibasis of $T$

(ii) If $1,2 \notin \Psi$, then $W \cup\left\{u_{1}, v_{s}\right\}$ is a multibasis of $T$

(iii) If $1 \notin \Psi$ and $2 \in \Psi$, then $W \cup\left\{u_{1}, v_{1}, v_{s}\right\}$ is a multibasis of $T$

Proof

(i) Assume that $1 \in \Psi$. By Case 1 in the proof of Proposition 6, it implies that $W \cup\left\{u_{1}\right\}$ is a multiresolving set of $T$. Hence, $\operatorname{dim}_{M}(T)=|\Psi|+1$ by Corollary 1 , and so $W \cup\left\{u_{1}\right\}$ is a multibasis of $T$.

(ii) Assume that $1,2 \notin \Psi$. By Case 2 in the proof of Proposition 6, it implies that $B=W \cup\left\{u_{1}, v_{s}\right\}$ is a multiresolving set of $T$. Therefore, $\operatorname{dim}_{M}(T) \leq$ $|\Psi|+2$. Next, we claim that $\operatorname{dim}_{M}(T) \geq|\Psi|+2$. Let $p=\min (\Psi)$. Since there are four components of $T-u_{p}$, it follows from Theorem 1 and Theorem 7 that at least one vertex from the component of $T-u_{p}$ containing a vertex $u_{p-1}$, belongs to every multiresolving set of $T$. Similarly, every multiresolving set must contain at least one vertex from the component of $T-u_{s-p+1}$ containing $u_{s-p+2}$. Therefore, by Observation 1, every multiresolving set of $T$ has cardinality at least $|\Psi|+2$. Hence, $\operatorname{dim}_{M}(T)=|\Psi|+2$ and so $W \cup\left\{u_{1}, v_{s}\right\}$ is a multibasis of $T$.

(iii) Assume that $1 \notin \Psi$ and $2 \in \Psi$. By Case 3 in the proof of Proposition 6, it implies that $W \cup\left\{u_{1}, v_{1}, v_{s}\right\}$ is a multiresolving set of $T$. Thus, $\operatorname{dim}_{M}(T) \leq|\Psi|+3$. Next, we show that $\operatorname{dim}_{M}(T) \geq|\Psi|+3$. Since there are four components of $T-u_{2}$, it follows by Theorem 7 that every multiresolving set of $T$ must contain at least one vertex of $\left\{u_{1}, v_{1}\right\}$. Similarly, every multiresolving set of $T$ must contain at least one vertex of $\left\{u_{s}, v_{s}\right\}$. We claim that every multiresolving set of $T$ contains three vertices of $\left\{u_{1}, u_{s}, v_{1}, v_{s}\right\}$. Suppose, contrary to our claim, that there is a multiresolving set $S$ of $T$ containing only one of $\left\{u_{1}, v_{1}\right\}$ and one of $\left\{u_{s}, v_{s}\right\}$. By Observation 1 , we may assume, without loss of generality, that $W \subset S$. If $u_{1}, u_{s} \in S$, then $\operatorname{mr}\left(u_{1} \mid S\right)=m r\left(w_{2} \mid S\right)$, which is impossible. If $u_{1}, v_{s} \in S$, then $m r\left(u_{1} \mid S\right)=m\left(w_{2} \mid S\right)$, a contradiction. If $v_{1}, u_{s} \in S$, then $m r\left(u_{s} \mid S\right)=m r\left(w_{s-1} \mid S\right)$, producing a contradiction. If $v_{1}, v_{s} \in S$, then $m r\left(v_{1} \mid S\right)=m r\left(v_{s} \mid S\right)$, which is also impossible. Therefore, every multiresolving set of $T$ has cardinality at least $|\Psi|+3$. Hence, $\operatorname{dim}_{M}(T)=|\Psi|+3$ and so $W \cup\left\{u_{1}, v_{1}, v_{s}\right\}$ is a multibasis of $T$.

Let $T$ be a symmetric caterpillar ca $\left(k_{1}, k_{2}, \ldots, k_{s}\right)$ with $k_{i}=1$ for some integer $i$ with $2 \leq i \leq s-1$. If $T-v_{i}$ is not a path, then by applying Theorems 5 and $8, \operatorname{dim}_{M}(T-$ $\left.v_{i}\right)=\operatorname{dim}_{M}(T)$. This observation provides us with the following more general result whose proof is straightforward and is therefore omitted.
Corollary 2. For $s \geq 3$, let $T$ be a symmetric caterpillar $c a\left(k_{1}, k_{2}, \ldots, k_{s}\right)$ with a second end-set $\Psi_{T}$ and let $T^{\prime}$ be a caterpillar ca $\left(l_{1}, l_{2}, \ldots, l_{s}\right)$ that is a subgraph of $T$ and is not a path, with a second end-set $\Psi_{T^{\prime}}$. If $\Psi_{T^{\prime}}=\Psi_{T}$, then $\operatorname{dim}_{M}\left(T^{\prime}\right)=\operatorname{dim}_{M}(T)$.

\section{Final Remarks}

A subdivision $T^{\prime}$ of a symmetric caterpillar $T$ is a graph that is obtained from $T$ by inserting vertices of degree 2 into some, all or none of the edges of $T$. It would be interesting to study the multibasis of the subdivision $T^{\prime}$ of $T$.

\section{Data Availability}

No data were used to support this study as no datasets were generated or analyzed during the current study. Other data sources are referenced throughout the paper.

\section{Conflicts of Interest}

The authors declare that they have no conflicts of interest.

\section{References}

[1] G. Chartrand, L. Eroh, M. A. Johnson, and O. R. Oellermann, "Resolvability in graphs and the metric dimension of a graph," Discrete Applied Mathematics, vol. 105, no. 1-3, pp. 99-113, 2000.

[2] P. J. Slater, "Leaves of trees," Congressus Numerantium, vol. 14 , pp. 549-559, 1988.

[3] P. J. Slater, "Dominating and reference sets in a graph," Journal of Mathematical and Physical Sciences, vol. 22, no. 4, pp. 445-455, 1988.

[4] B. L. Hulme, A. W. Shiver, and P. J. Slater, FIRE: A Subroutine for Fire Protection Network Analysis, SAND 81-1261, Sandia National Laboratories, Albuquerque, NM, USA, 1981.

[5] B. L. Hulme, A. W. Shiver, and P. J. Slater, Computing Minimum Cost Fire protection, SAND 820809, Sandia National Laboratories, Albuquerque, NM, USA, 1982.

[6] B. L. Hulme, A. W. Shiver, and P. J. Slater, "A boolean algebraic analysis of fire protection," in Algebraic and Combinatorial Methods in Operations Research, Proceedings of the Workshop on Algebraic Structures in Operations Research, pp. 215-227, Elsevier, Amsterdam, Netherlands, 1984.

[7] F. Harary and R. A. Melter, "On the metric dimension of a graph,” Ars Combinatoria, vol. 2, pp. 191-195, 1976.

[8] M. Johnson, "Browsable structure-activity datasets," Advances in Molecular Similarity, vol. 2, pp. 153-170, 1999.

[9] S. Khuller, B. Rsghavachari, and A. Rosenfeld, Localization in Graphs, CS-TR-3326, University of Maryland, Riverdale Park, MD, USA, 1994.

[10] V. Saenpholphat, "On multiset dimension in graphs," Academic SWU, vol. 1, pp. 193-202, 2009.

[11] V. Khemmani and S. Isariyapalakul, "The multiresolving sets of graphs with prescribed multisimilar equivalence classes," International Journal of Mathematics and Mathematical Sciences, vol. 2018, Article ID 8978193, 6 pages, 2018.

[12] V. Khemmani and S. Isariyapalakul, "The characterization of caterpillars with multidimension 3," Thai Journal of Mathematics, pp. 247-259, 2020.

[13] R. Simanjuntak, T. Vetrík, and P. B. Mulia, "The multiset dimension of graphs," 2017, https://arxiv.org/abs/1711.00225. 